\title{
A BYPASS FOR THE INSTITUTIONAL REVIEW BOARD: REFLECTIONS ON THE CLEVELAND CLINIC STUDY OF THE BATISTA OPERATION*
}

\author{
Martin F. McKneally MD, PhD
}

W hen I heard the oral presentation of the study of the Batista operation conducted by Patrick McCarthy and his colleagues last May, I shared in the thrill that was palpable in the audience. Chosen by the program committee as one of the first presentations at the meeting of The American Association for Thoracic Surgery, the study delivered a clear and practical surgical judgment about the validity of left ventricular reduction, a new procedure widely regarded as radically innovative.

We learned that the investigators removed part of the ventricle in patients judged ill enough to have their whole heart removed and replaced. The physiological effects of remodeling the heart were clearly described. Best of all, the operation was performed in a setting that could offer the patients a safe fallback procedure, either mechanical assistance or transplantation.

It seemed an ideal way to "try the treatment out," testing its efficacy and appropriateness. McCarthy and his colleagues had built a reliable safety net for their patients, which would protect them from the substantially higher mortality associated with the operation in other settings.

When the manuscript describing The Cleveland Clinic study reached the Journal's reviewers, it prompted them to ask several questions that focused on ethical issues: (1) Were the patients sufficiently informed about the study? (2) Shouldn't the study have been reviewed by a research ethics board such as the Institutional Review Board (IRB) of The Cleveland Clinic? (3) What is the Journal's role in setting or enforcing ethical standards of patient care and research?

Our Editor, Andrew Wechsler, asked me to comment on some of the ethical issues and to provide a definition of ethics. I will begin with the definition: Ethics is a plural noun, ${ }^{1}$ referring to various systems of principles

\footnotetext{
*For related article, see p. 879.

From the Department of Surgery and Joint Centre for Bioethics, University of Toronto, Toronto General Hospital, University Health Network, Toronto, Ontario, Canada.

J Thorac Cardiovasc Surg 2001;121:837-9

Copyright (C) 2001 by The American Association for Thoracic Surgery

$0022-5223 / 2001 \$ 35.00+0 \quad \mathbf{1 2 / 1 / 1 1 2 5 2 1}$

doi: $10.1067 / \mathrm{mtc} .2001 .112521$
}

and values that govern the behavior of particular cultures, groups, or individuals. In short, an ethic is a system of beliefs about "what we should do." While religious ethics are grounded in a single coherent view of values based on a divine perspective, ${ }^{1}$ secular ethics, such as the ethic of surgery, are socially constructed. This means that members of the group (in our case, surgeons) come to an agreement about the values and principles that will govern our behavior. Surgeons and their surgical journals are then obliged to conduct themselves in accordance with those values and principles.

Let us now look at the issues raised by our reviewers.

1. Were the patients sufficiently informed about the study? Reviewers raised concerns about whether they should consider this article as a report of an observational study or an experiment. Observational studies report on the outcomes of treatment as chosen by physicians and patients. The disclosure of information required for consent to treatment is generally less than that required for experiments. Experiments require special protections for patient-subjects, such as formal consent to be studied as well as treated, approval of the study protocol by an independent research ethics board, and, occasionally, oversight by a safety monitoring mechanism to protect the participants from potential harms that can befall both investigators and patients. ${ }^{2}$ Participants in an experiment carry an increased risk of having their best interests subordinated to a protocol that requires systematic application and evaluation of the treatment. Because such studies commit patients and their physicians to follow an assigned treatment program, their freedom is restricted. The term "crossing over" underlines this constraint; a change in treatment during a study violates a boundary placed around the choices imposed by the study protocol. The protocol limits choices for a good reason-to generate data that can be analyzed to produce generalizable knowledge about the treatment. Did McCarthy and his colleagues limit their patients' choices more than any practicing surgeon limits choices when performing an operation, thus committing those patients to a defined treatment? If so, the patients should be made aware of the limits imposed by the protocol.

2. Should this study be reviewed by a research ethics board such as the IRB of The Cleveland Clinic? What was troubling our reviewers, I think, is not so much the 
systematic, somewhat protocol-driven limitation on treatment options. I suspect they were more troubled by the fact that the Batista operation is a nonvalidated procedure. $^{2,3}$ Cardiologists and surgeons did not know the answers to such questions as these: Does this operation really work? Which patients will benefit? When should the operation be applied in the course of the illness?

Every application of a validated treatment to a particular patient is a quasi-experiment, to see how the treatment works in that particular patient. At some level, patients know that we are "trying it out" to see if the treatment will work well for them. However, they have some assurance that the treatment usually works reasonably well in patients with illness similar to theirs and is accepted as a valid treatment by informed members of the professional community.

In The Cleveland Clinic study, the treatment was also on trial. When the experiment involves a nonvalidated treatment, the constraint of a protocol may be intensified. When the staff become as keen to learn about the benefits, harms, and other unknown aspects of a treatment as they are to know how it worked in any one of the patients they are caring for, a competition of interests arises. The patients are at risk of being used instrumentally to gain answers to a series of general questions that interest the investigators and, among others, The American Association for Thoracic Surgery Program Committee and the readers of this Journal. This risk, and the risk of experiencing unknown effects of the treatment, are among those that IRBs require researchers to disclose in the process of informing and obtaining consent from research subjects.

The IRB was developed to bring greater safety, transparency, and public defensibility to the process of conducting experiments in human patients. The participation of lay members, chaplains, lawyers, and nurses, as well as physicians, provides a broader perspective of public oversight than that provided by community representatives on the institutional board of trustees. The IRB's experience with research protocols facilitates a systematic approach to thinking about and protecting the interests of participants in research. The IRB advises the professional and administrative staff of the institution only when it is requested to do so or when it is required by defined institutional policy to give an opinion. These requests and requirements are generally triggered by concerns of the professional staff about an experimental approach to patient care that is deemed to be outside the boundary that defines the usual and customary treatments offered to patients. This boundary is fairly clear in pharmaceutical research, where the pharmacopoeia of approved drugs and the procedures for testing unap- proved drugs are defined in the United States by the federal Food and Drug Administration. The threshold for requesting the advice of the IRB is not conspicuously marked in the domain of surgical innovations. Surgical procedures evolve gradually; surgeons modify techniques and incorporate new materials or devices that show promise of giving a better outcome as a routine part of their practice. The boundary between evolution of validated treatments and the introduction of experimental nonvalidated treatment is neither well defined nor stable over time. Since IRBs have finite resources of time, personnel, and technical knowledge, their oversight of surgical innovation has natural limits. When should the advice of this additional institutional safeguard be requested? When should it be required? The answer to these questions is usually worked out at the local level, with somewhat haphazard results.

3. What is the Journal's role in setting or enforcing ethical standards of patient care and research? The Journal provides a forum for publication of important information. When the Journal publishes a manuscript, it gives implicit approval of the scientific and ethical value of the work. The ethical standards are based on the World Medical Association's Declaration of Helsinki, Recommendations Guiding Medical Doctors in Biomedical Research Involving Human Subjects. ${ }^{4}$ The Helsinki declaration sets clear requirements, including approval by an independent committee, for experimental studies. It is less clear on new therapeutic measures. Although their use is accepted "if it offers hope of saving life, reestablishing health, or alleviating suffering," combining research with professional care for the acquisition of new medical knowledge is circumscribed. "The physician can combine medical research with professional care [for] the acquisition of new medical knowledge only to the extent that the . . . research is justified by its potential . . . therapeutic value for the patient" [emphasis mine]. ${ }^{4}$ This standard is reasonable and patient centered, but it provides insufficient guidance for answering our reviewers' questions. The position of the Journal, generally based on these principles, requires refinement and specification. Nonvalidated procedures and devices "that offer hope ..." are proliferating and entering practice at an unprecedented rate in this exciting era of surgical innovation in our specialty.

I will open the dialogue with readers that is needed to refine our policy by taking a position. I favor a journal policy that continues the requirement for IRB approval of experimental studies with well-defined protocols that can be explained in advance to an IRB and followed precisely to validate a research hypothesis. I 
would propose a second mechanism of approval for the systematic study of nonvalidated surgical procedures and devices that recognizes the unique features of studies that attempt to answer the question, "How can we make this work?" Surgical intuition, unspecifiable in advance, is pivotal to adapting and modifying new procedures. ${ }^{5}$ This unique particularity is readily understood by surgeons, but it perplexes thoughtful people outside surgery, including many IRB members.

My proposal draws in part on the experience of using research protocols approved by larger agencies such as the cooperative oncology groups. These agencies provide trustworthy background information and templates for research protocols and consent forms. This process helps to relieve the burden of reviewing unknown areas beyond the expertise of a local committee. An agency like the Joint Society of Thoracic Surgeons/American Association for Thoracic Surgery Ad Hoc Committee on New Technology Assessment might review and approve a surgeon's protocol for evaluating a new procedure. A consent form template emphasizing novelty, the learning curve, the inevitability of procedure modifications, planned safety monitoring, and timely reporting would provide the flexibility needed to navigate uncharted waters, as McCarthy and his colleagues did so well.* The

*When I talked with Dr. McCarthy about his approach to the ethical issues discussed here, I learned about the unique environment in which his research is conducted. Although the authors did not seek formal approval from the IRB for this study, there is close collaboration and communication with IRB members and a nuanced understanding of research ethics among the investigators, one of whom is a former IRB chairman.
Journal could reasonably accept studies that followed these protocols without requiring prior approval by the local IRB. I believe local institutional administrators, practitioners, patients, and the public would view this bold initiative as evidence that we as a profession are adapting and constructing our surgical ethic with integrity and sensitivity to the challenges of evolving surgical technology.

This editorial is an invitation to our readers to provide their guidance to the Journal and the profession as the ethic of surgery evolves. I will summarize the suggestions you send with appropriate acknowledgment in a subsequent editorial comment.

Received for publication Aug 11, 2000; revisions requested Aug 22, 2000; revisions received Sept 12, 2000; accepted for publication Sept 15, 2000.

Address for reprints and suggestions: Martin F. McKneally, MD, 77 Forest Grove Dr, Toronto, Ontario, Canada M2K 1Z4 (E-mail: martin.mckneally@utoronto.ca).

\section{REFERENCES}

1. Engelhardt HT Jr. The foundations of bioethics. 2nd ed. New York: Oxford University Press; 1996. p. 3-31.

2. Levine RJ. Ethics and regulation of clinical research. 2nd ed. New Haven: Yale University Press: 1988. p. 4-5, 127-30.

3. McKneally MF. Ethical problems in surgery: innovation leading to unforeseen complications. World J Surg 23;1999:786-8.

4. World Medical Association's Declaration of Helsinki, Recommendations Guiding Medical Doctors in Biomedical Research Involving Human Subjects. JAMA 1997;277:925-6 (available online at www.vitreoussociety.org/journal/instruct/ helsinki.htm).

5. Abernathy CM, Hamm RM. Surgical intuition: What it is and how to get it. Philadelphia: Hanley \& Belfus; 1995. p. 23-7.

\section{Bound volumes available to subscribers}

Bound volumes of The Journal of Thoracic and Cardiovascular Surgery are available to subscribers (only) for the 2001 issues from the Publisher, at a cost of \$134.00 for domestic, \$165.85 for Canadian, and \$155.00 for international subscribers for Vol 121 (January-June) and Vol 122 (July-December). Shipping charges are included. Each bound volume contains a subject and author index and all advertising is removed. Copies are shipped within 60 days after publication of the last issue of the volume. The binding is durable buckram with the Journal name, volume number, and year stamped in gold on the spine. Payment must accompany all orders. Contact Mosby, Subscription Customer Service, 6277 Sea Harbor Dr, Orlando, FL 32887,USA; phone 800-654-2452 or 407-345-4000.

Subscriptions must be in force to qualify. Bound volumes are not available in place of a regular Journal subscription. 